\title{
Reinstatement: An attempt at replication
}

EDWARD E. SHUBAT and JAMES M. WHITEHOUSE ${ }^{2}$ University of Montana, Missoula, Mont. 59801

An attempt was made to replicate Campbell \& Jaynes' (1966) demonstration of the reinstatement effect. The replication did find effects attributable to reinstatement. However, inconsistencies between the two experiments were sufficient to question the pervasive importance originally attributed to the concept of reinstatement. It was suggested that age, shock level, and their interaction be fully explored.

Campbell \& Jaynes (1966) have proposed a new mechanism, reinstatement, which they regard as important in maintaining the effects of early experience upon adult behavior.

"By reinstatement we denote a small amount of partial practice or repetition of an experience over the developmental period which is enough to maintain an early learned response at a high level, but is not enough to produce any effect in animals which have not had the early experience." (Campbell \& Jaynes, 1966). Their first demonstration of this effect appears simple and makes use of a common instance of learning. A shuttle box is divided, the black side having a grid floor and the white, other side, a solid floor. Wistar rats, when given a fear experience (shock) at approximately 25 days of age demonstrate a significant avoidance of the grid when tested at approximately 54 days of age, if they have received one reinstatement trial per week until the time of the test. Animals without either the early fear experience or the reinstatements do not demonstrate this avoidance and prefer the grid side.

From these results they extend the possibility that reinstatement is of pervasive importance for ontogeny of behavior in vertebrates.

"In a general sense, we propose that any learned response, whether acquired in infancy or adulthood, conscious or unconscious, instrumental or autonomic, joyful or traumatic, can be maintained at a high level by an occasional reinstatement." (Campbell \& Jaynes, 1966).

An effect of this importance must be replicable by other investigators. Consequently, an attempt to replicate this experiment was advanced with these goals: (a) To replicate the reinstatement effect, (b) gather baseline data for further research within the fear reinstatement paradigm, (c) add a control group to strengthen the design. The new control group was felt to be important since it would provide a true baseline from which the reinstatement effect could be assessed. Campbell \& Campbell (1962) report their control groups demonstrated a preference for the grid side after 30-40 days of age. However, these animals had been through the same procedure as the experimental animals though they had not been shocked. It was felt that a control group completely naive to the apparatus prior to the $1 \mathrm{~h}$ preference test at approximately 54 days of age would allow an assessment of any procedural effects in former control groups.

\section{METHOD}

The Ss were 40 Wistar rats shipped at 20 days of age from Simonsen Laboratories. At 25 days of age, these animals were divided into four groups with equal numbers of each sex. Groups 1-3 were treated with the same methodological procedures described by Campbell \& Jaynes (1966). Group 4 was the new control group and given the $1 \mathrm{~h}$ preference test when approximately 58 days of age and completely naive to the apparatus.

Dimensions, color and grid construction were those specified by Campbell \& Jaynes (1966). Construction material was changed to Plexiglas. The tilting floor was improved by supporting it on needle bearings constructed in the shop, and zero balanced by a sliding counterweight. The mercury well switch was discarded in favor of a Hunter photocell relay which was energized by slight grid movement interrupting the light source. Sound insulation was improved by placing the box within a converted biological culture oven which was quite well insulated and had both inner and outer doors. White noise was generated and distributed throughout the experimental research building. All control and shock apparatus was isolated in another room and connected to the box by umbilicals. Silenced Hunter Klockcounters were used for measuring time spent in each compartment in $12 \mathrm{~min}$ segments. Illumination was provided by a $7 \mathrm{~W}$, soft white bulb mounted in a light diffuser and wired in series with a $15 \mathrm{~W}, 2250 \mathrm{ohm}$ resistor. Shock was supplied by an Applegate Model 250 Stimulator, which is a constant current ac source with an upper limit of $5 \mathrm{~mA}$, and was not the "matched impedance ac" circuit of Campbell and Jaynes. A Davis grid scrambler was used. These changes were instituted in order to specify standard laboratory equipment readily available to all investigators. Groups 1-3 were expected to replicate the reinstatement experiment while Group 4 was expected to demonstrate preference for the grid side of the apparatus.

\section{RESULTS}

Group 1 (fear, reinstatements) spent a mean total time of 53 $\min 8 \mathrm{sec}$ in the solid floor side of the apparatus (see Fig. 1). Campbell \& Jaynes (1966) regard such behavior as evidence for retention of fear and representative of the reinstatement effect. An attempt to make a direct comparison of these results with the original experiment was precluded because the original data was not readily available (Campbell, 1967). By inspection, it does appear that the animals within this group demonstrate an even stronger preference for the solid floor than did those in the original experiment. The shape of the two curves compare favorably.

Group 2 (fear, no reinstatements) demonstrated learned avoidance by spending a mean total time of $29 \mathrm{~min} 41 \mathrm{sec}$ on the solid floor. However, the avoidance performance was clearly inferior to Group 1, and was not maintained at a high level. However, this replication group shows superior avoidance when compared to Campbell and Jaynes's group for they averaged approximately 19 more minutes on the solid floor. It is also apparent that this group's curve is different from that reported in

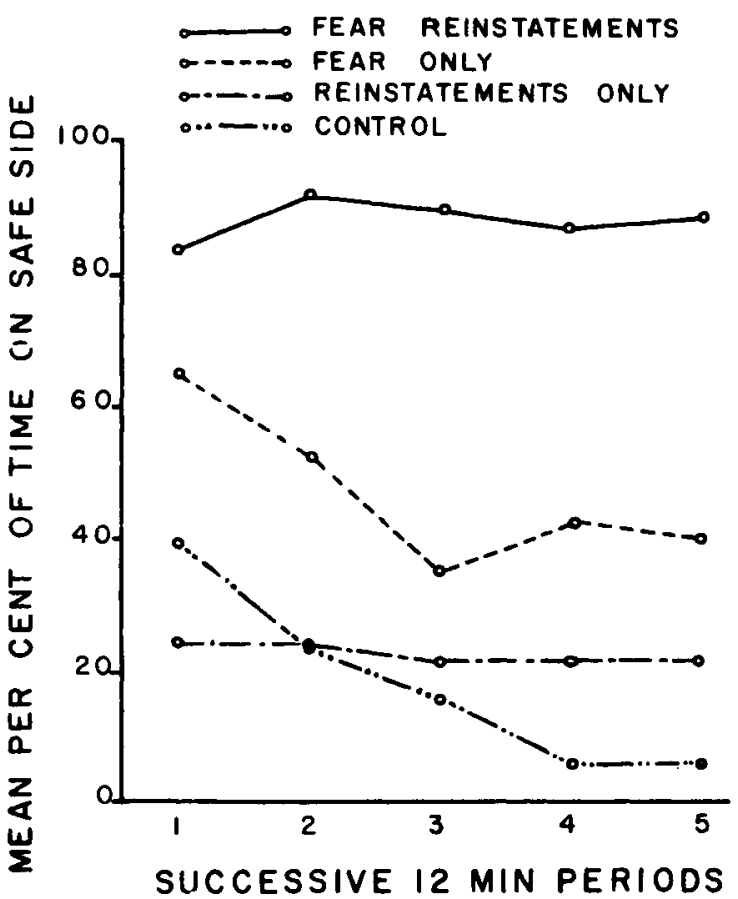

Fig. 1. Effect of reinstatement. 
the original experiment; it is not flat but negatively accelerated (see Fig. 1).

Group 3 (no fear, reinstatements) spent a mean total time of 14 min $18 \mathrm{sec}$ on the solid floor and the curve has a flat appearance found in the original experiment. The concept that the reinstatements alone produce no effect was supported.

Group 4 (naive controls) spent a mean total time of $10 \mathrm{~min} 9$ sec on the solid floor. This is a demonstration of a definite preference for the grid side and supports the similar finding found for less naive control groups (Campbell \& Campbell, 1962; Campbell \& Jaynes, 1966). Note, however, that this group has a negatively accelerated curve.

As expected, Group 1 is significantly different from all other groups $(\mathrm{p}<.01$, Mann-Whitney $U$ test $)$. However, Group 2 is also significantly different from all other groups $(\mathrm{p}<.01)$ and except for a difference from Group 1, would not be predicted from the original experiment. As stated before, some ranges and curve shapes appear to be altered.

\section{DISCUSSION}

Though differences were noted, this replication did support the concept of a reinstatement effect. This attempt was not hampered by "freezing" behavior in Ss that has concerned other investigators (Campbell, 1967).

An ad hoc analysis was undertaken to locate the possible source of variation. It is not likely that the differences were a direct consequence of changes in construction material, grid balance refinement, circuit actuation, light specification and sound isolation. These changes did add to equipment clarification, refine measurement of the dependent variable but were not a drastic departure from the original experiment.

Examination of the original experiment and its antecedents (Campbell \& Teghtsoonian, 1958), (Campbell \& Campbell, 1962) seem to pinpoint the shock source as the most important variable and this was the major departure in apparatus selection for this replication. The Applegate stimulator was selected for its constant current ac circuit and it is readily available to all investigators. It was found, however, to be partially responsible for a spuriously high shock when in the scrambler circuit due to high voltage transients. Fortunately, the transients were of very brief duration and the use of constant current ac mitigated against fatal shock or tetenization.

Another source of difference may lie in the difficulty of determining the exact shock level used by Campbell \& Jaynes (1966). They state $170 \mathrm{~V}$ is the shock level but one must go to Campbell \& Campbell (1962) to discover this voltage is measured across the secondary of a step-up transformer and prior to a $150.000 \mathrm{ohm}$ resistor in series with the rat. The use of $170 \mathrm{~V}$ as a shock level description is misleading. One $\mathrm{mA}$ was calcualted as the appropriate level when measured as a short circuit across the grid for this replication. However, if one uses the decibel as a common logarithmic scale to compare the two sources of shock (Campbell \& Tghtsoonian, 1958) the difference between these sources is clarified. Campbell \& Jaynes (1966) use a shock level approximately $15 \mathrm{~dB}$ above a $0 \mathrm{~dB}$ level while the $1 \mathrm{~mA}$ shock is approximately $24 \mathrm{~dB}$ above a 0 level.

Albeit ad hoc analysis, it does appear likely the differences between the two experiments were predominantly due to a spuriously high shock level and shock source differences. Support for this position is furnished by Campbell \& Campbell's (1962) report, that young rats demonstrated a longer retention interval of fear in a conditioned response suppression paradigm when a $300 \mathrm{~V}$ (approximately $20 \mathrm{~dB}$ ) shock was used. Campbell \& Teghtsoonian (1958) report differential behavior effects from shock source differences.

Group 4 demonstrated an expected preference for the grid side of the apparatus. This curve is not flat as is that of the usual experimental controls. It is probable that procedural familiarity with the apparatus flattens the curve and the importance of this effect is unknown.

It is clear that one must attend carefully to the shock source and level if the fear reinstatement paradigm is to be replicated. Further, some questions about the fear reinstatement effect should be raised. Is this effect dependent upon age, level of shock, or an interaction of these two factors? It is not likely that the effect can be demonstrated with the present level of shock in the adult animal since their retention interval for a similar fear experience is much longer (Campbell \& Campbell, 1962). The necessity for reinstatement may vary as a function of the level of induced fear. Its importance for the maintenance of an early learned response may be minimal if the original experience is severe.

\section{REFERENCES}

CAMPBELL, B. A. Private communication, 1967.

CAMPBELL, B. A., \& CAMPBELL, E. H. Retentions and extinction of learned fear in infant and adult rats. Journal of Comparative \& Physiological Psychology, 1962, 55, 1-8.

CAMPBELL, B. A., \& JAYNES, J. Reinstatement. Psychological Review, $1966,73,478-480$.

CAMPBELL, B. A., \& TEGHTSOONIAN, R. Electrical and behavioral effects of different types of shock stimuli on the rat. Journal of Comparative \& Physiological Psychology, 1958, 51, 185-192.

\section{NOTE}

1. This research was supported in part by Public Health Service Predoctoral Research Fellowship MH-36,891-01 from National Institute of Mental Health. 2. Now at Drake University, Des Moines, lowa 50311 